\title{
Storage and discharge of a granular fluid
}

\author{
Hector Pacheco-Martinez, ${ }^{1}$ Henk Jan van Gerner, ${ }^{2}$ and J. C. Ruiz-Suárez,* \\ ${ }^{1}$ Departamento de Física Aplicada, CINVESTAV-Mérida, A. P. 73 Cordemex, Mérida, Yucatán 97310, México \\ ${ }^{2}$ Faculty of Science and Technology, University of Twente, P.O. Box 217, 7500 AE Enschede, The Netherlands \\ ${ }^{3}$ CINVESTAV-Monterrey, Autopista Nueva al Aeropuerto Km. 9.5, Apodaca, Nuevo León 66600, México \\ (Received 19 July 2007; revised manuscript received 27 November 2007; published 12 February 2008)
}

Experiments and computational simulations are carried out to study the behavior of a granular column in a silo whose walls are able to vibrate horizontally. The column is brought to a steady fluidized state and it behaves similar to a hydrostatic system. We study the dynamics of the granular discharge through openings at the bottom of the silo in order to search for a Torricelli-like behavior. We show that the flow rate scales with the wall induced shear rate, and at high rates, the granular bed indeed discharges similar to a viscous fluid.

DOI: 10.1103/PhysRevE.77.021303

PACS number(s): 45.70.Mg, 83.80.Fg

\section{INTRODUCTION}

One of the most conspicuous laboratories in granular physics is a silo full of grains. If the column inside the silo is in repose, it behaves as a strange solid: the grain-grain interactions break up the symmetry imposed by gravity and a screening effect appears, redirecting most of the weight of the column onto the side walls of the silo (Janssen effect [1]). This, of course, is an annoying phenomenon for a farmer; not only due to the impossibility of knowing the real mass the silo holds, but because the walls might not sustain the normal forces applied by the grains. If we now discharge the silo through a hole made at its bottom, the material flows, but it behaves as a strange fluid. Even if the diameter of the aperture is larger than the size of the grains, clogging structures appear interrupting the discharge (jamming effect $[2,3]$ ). Due to the confluence of these two phenomena, and the importance they have in industrial silos and hoppers, granular storage and discharge have been studied profusely over the decades.

The Janssen effect states that the mass measured at the bottom of the silo is not the real mass $M$ one pours into it, but an apparent one that follows the relation $M_{\text {app }}=M_{s}(1$ $\left.-e^{-M / M_{s}}\right)$; where $M_{s}$ is the mass measured at the bottom of the silo when saturation is reached. This effect was observed by Janssen at the end of the 19th century, but it is still a matter of study nowadays. Indeed, well controlled experiments have been carried out recently in laboratory silos [2-4]. Also, it has been demonstrated that the Janssen law is valid even if the side walls move vertically [5].

Grain discharge from a silo was first studied by engineers (see the review of Nedderman et al. [6]). Within that community, in 1960 Beverloo and co-workers proposed, after some systematic experiments changing several variables, the formula used today to correlate the outflow rate $W$ with the diameter $D$ of the aperture [7]: $W=\zeta \rho_{B} g^{1 / 2}(D-k d)^{5 / 2}$, where $\zeta$ and $k$ are constants, $g$ is the acceleration of gravity, and $\rho_{B}$ the effective density of the granulate. While this expression is empirical, it can be also deduced from basic dimensional considerations [6].

\footnotetext{
*cruiz@mda.cinvestav.mx
}

Two important behaviors are observed during the discharge. First, in order to obtain a continuous flow, the diameter of the outlet has to be greater than a critical diameter $D_{c}$ (if the grains are spherical, the value of $D_{c}$ lies between 4 and 5 times the diameter of a single grain $d$ ). Secondly, the discharge rate is constant and independent of the column height $H$ (usually called the head). This second phenomenon is puzzling, because it contrasts with the case of a normal liquid flowing out from a container, where the flux $W$ decreases as $H$ decreases (if the liquid has very low viscosity, $W$ varies according to Torricelli's law: $W$ is proportional to $\left.H^{1 / 2}\right)$.

Despite some unsolved concerns in the behavior of dense granular flows, the extended belief in the literature is that such flows are governed by the rapid formation of stressbearing structures due to interparticle interactions. Furthermore, since grains are inelastic, the rather fast collapse into such structures imposes a time scale. When this scale is shorter than the scale of the time imposed by the strain rate in the system (normally the case in gravity-driven flows), the grains move coherently [8].

Is the formation of such structures near the hole of a silo, and the velocity correlation of the grains, beneath the constant discharge rate in a silo? A plausible strategy to advance the understanding of this problem is to inject energy in the system. In fact, by shearing the column and fluidize it, we could overcome the formation of arches and learn about the effect they have in the discharge dynamics. However, we have to inject energy to the core of the column on a time scale shorter than the one imposed by grain inelasticity. To our knowledge, only few works have studied the discharge rates in vibrating hoppers, silos, or hourglasses [9-12]. However, in all these cases, the containers were shaken either vertically or horizontally as whole units.

Our experimental setup is different. We constructed a silo able to fluidize the entire column in steady-state conditions with no convection. The aim of the present work is to study the discharge of such steady-state vibro-fluidized granular column. We complement our experimental study with 3D computer simulations.

\section{SETUP}

The silo is a segmented container composed of eight independent acrylic panels, see Fig. 1. The panels, with dimen- 


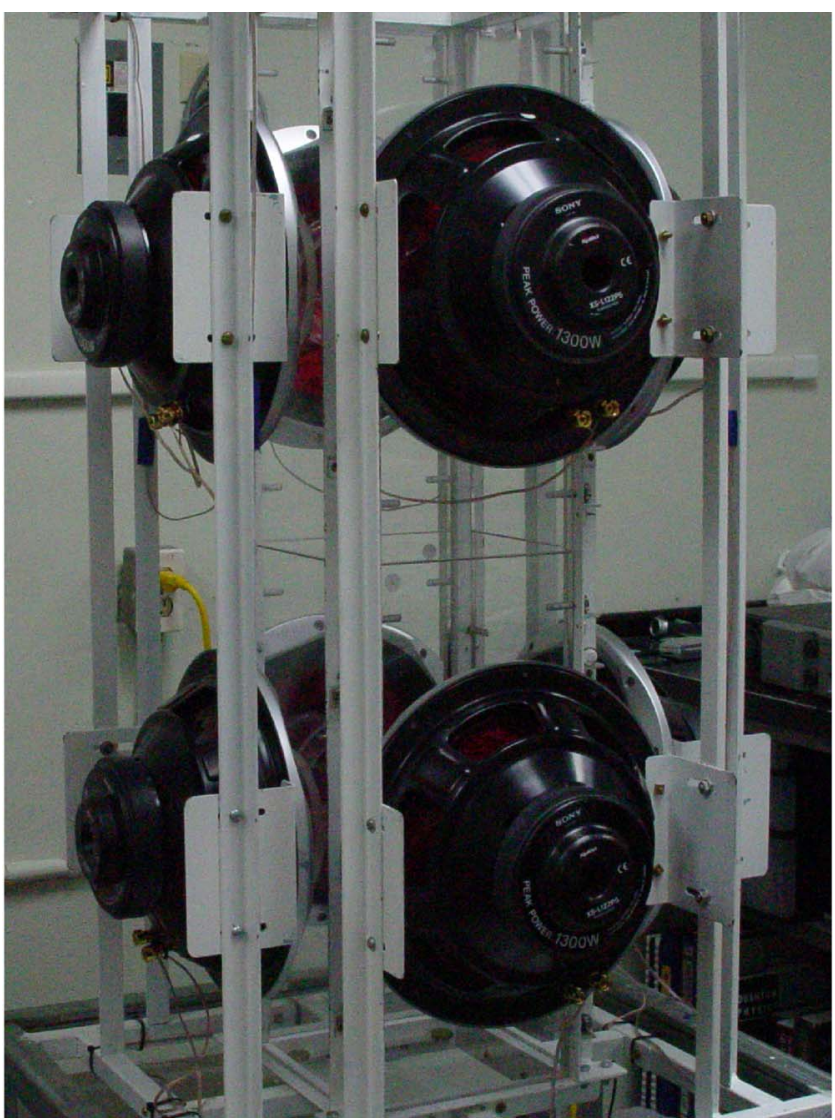

FIG. 1. (Color online) Photograph of the real silo. See the text.

sions $1 \times 20 \times 50 \mathrm{~cm}$, have at their corners $1 \mathrm{~cm}$ diameter steel bearings. These bearings have the function of supporting the weight of the panels, allowing as well the possibility of back-and-forth small movements. Each one of the bearings rest on metal pieces soldered onto a heavy metal structure. The panels are firmly attached to power speakers, these are also fixed to the structure and are connected in parallel, fed by a high-power amplifier (EG-4000) connected to a function generator (HP-33120A). Two panels, one on top of the other, form a wall. Therefore, the silo has $1 \mathrm{~m}$ of height and $400 \mathrm{~cm}^{2}$ of cross section. Neighboring walls move out of phase (while two opposite walls move in, the others move out). In doing this, we maintain the total volume of the granular column constant. The panels do not touch each other (the space left between them and the vertical beams of the structure is $1 \mathrm{~mm}$ ). A similar wall-moving container has been used by us elsewhere to fluidize a granular bed and prove Archimedean buoyancy [13]. We use very light particles (polystyrene spheres of density $16 \mathrm{~kg} / \mathrm{m}^{3}$ ) to fill the silo. The mean diameter of the spheres is $4.65 \mathrm{~mm}$ with a friction coefficient of $0.4 \pm 0.1$. We have found, by using a fast camera at $2000 \mathrm{fps}$, that the restitution coefficient of these particles in air is $0.8 \pm 0.1$. The maximum peak-to-peak vibration amplitude of the walls is $3 \mathrm{~mm}$. In order to avoid static charges, the spheres were treated with an antistatic spray, although some times few of them were attracted to the walls.

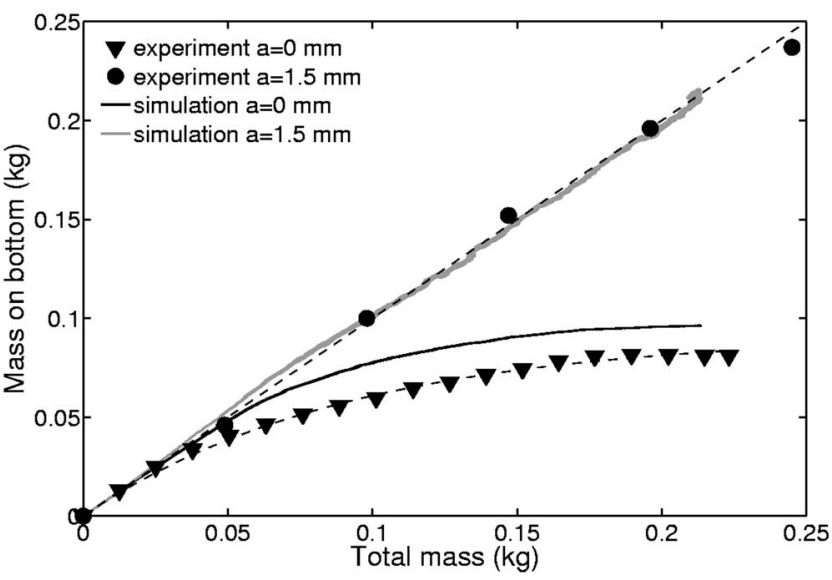

FIG. 2. Plot of the mass measured at the bottom of the silo as a function of the mass poured into it. The triangular points were taken using static walls. The circular points were obtained experimentally with vibrating walls. With static walls, the measured mass follows Janssen's law, which is indicated by the dashed curved black line. With vibrating walls, a hydrostatic behavior is obtained, which is indicated by the dashed straight black line. The dark gray line is obtained in a simulation with vibrating walls. The light gray line is obtained using static walls. Both the total mass and the mass on the bottom obtained in the simulation are multiplied by five for comparison with the experimental results.

\section{EXPERIMENTAL RESULTS}

In our first experiment, particles were slowly poured into the silo. The bottom of it, an acrylic panel also separated from the lateral walls and the structure, was fixed to a balance. During the filling process, the function generator is turned off. In order to keep the walls firmly fixed, the 32 roll-bearings were glued onto the small bases they roll. In Fig. 2 we plot the mass $M_{\text {app }}$ (measured with the balance) versus the real mass $M$ poured into the silo. We obtained the expected Janssen's effect. Next, we empty the silo and repeat the pouring process. This time, however, the walls are put to vibrate at a frequency of $20 \mathrm{~Hz}$ and amplitude of $1.5 \mathrm{~mm}$ (previously, the glue on the bearings was removed). The data are also plotted in Fig. 2. Instead of an exponential curve, a straight line $M_{\text {app }}=M$ is obtained. This result indicates that Janssen's effect vanishes and the entire mass of the column is received by the bottom. Since this is what occurs in standard liquids, the granular fluidized column behaves, apparently, similar to a hydrostatic system.

It is well known that Janssen's effect vanishes in silos with frictionless walls. Indeed, in such ideal conditions stress paths do not "anchor" at the walls and the bottom of the silo receives the entire weight of the column. But the system is statically inert in that case, not hydrostatic. In our vibrating silo the weight of the column is not only borne entirely by the bottom but, in addition, the particles jiggle around their equilibrium positions. We carried out a simple experiment to grasp the nature of this steady dynamics. We introduced into the bed a hollow spherical intruder made of polystyrene (effective density of $6 \mathrm{~kg} / \mathrm{m}^{3}$ and diameter $12 \mathrm{~cm}$ ). We observe that the intruder rises to the surface. Since there is no con- 


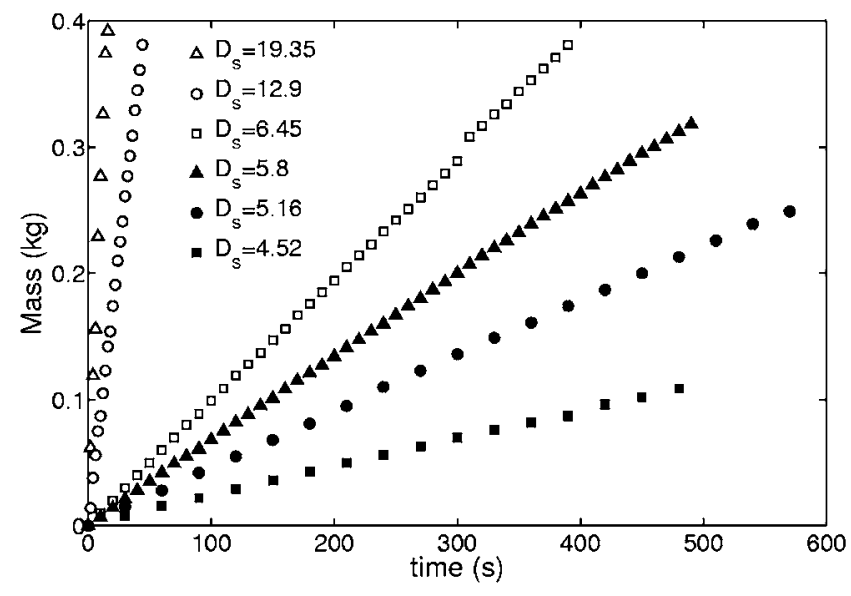

FIG. 3. A silo discharge experiment. The mass leaving the silo is plotted as a function of time, for different scaled outlet diameters $(f=20 \mathrm{~Hz}, a=1.5 \mathrm{~mm})$. The linear behavior means that the flow rate is constant.

vection [14], air drag [15], or inertia [16] (essentially because there is no vertical vibration applied to the silo), the only mechanism to segregate the intruder to the top is Archimedean buoyancy. Buoyancy is a physical effect due to the hydrostatic pressure acting on an intruder inside a fluid (there is more pressure on the lower side of the intruder than above it) and this is precisely the physical meaning of the straight line plotted in Fig. 2. The reader can find a thorough discussion on this subject in our previous work [13].

We now discharge the silo through a hole made at its bottom and measured the out flowing mass as a function of time. We do this for several holes, always with diameters larger than $D_{c}$ to avoid jamming. First, we discharge the silo with still walls. The obtained straight lines (see Fig. 3) confirm that the outflow is constant in time. Moreover, the slopes of these lines, as a function of $D$, conform to the power law found by Beverloo (see Fig. 4).

Finally, we discharge the silo while the walls vibrate. We have shown that this special shearing is able to continuously break the arches at the walls, producing hydrostaticlike conditions (at least from the point of view of Buoyancy). However, will it modify the way the silo discharges? Under the above shearing conditions ( $20 \mathrm{~Hz}$ and amplitude of $1.5 \mathrm{~mm}$ ) the answer is that it does not. The results are identical to the ones plotted in Fig. 3.

An important difference is, nevertheless, observed: when the walls are still, a funnel on the free surface of the head is formed, more profound at the end of the discharge, indicating that the particles in the core of the column descend faster than particles near the walls. However, when the walls vibrate, the funnel on the surface does not form and we observe instead a descending flat head. This, of course, can be easily explained: due to the vibration, the friction near to the walls decreases, the particles descend faster and, therefore, the head remains flat.

Summarizing, the Janssen's effect disappears when the walls vibrate, however, despite the column is fully fluidized, the discharge dynamics still satisfies Beverloo's scaling (although the free surface morphology of the head changes).

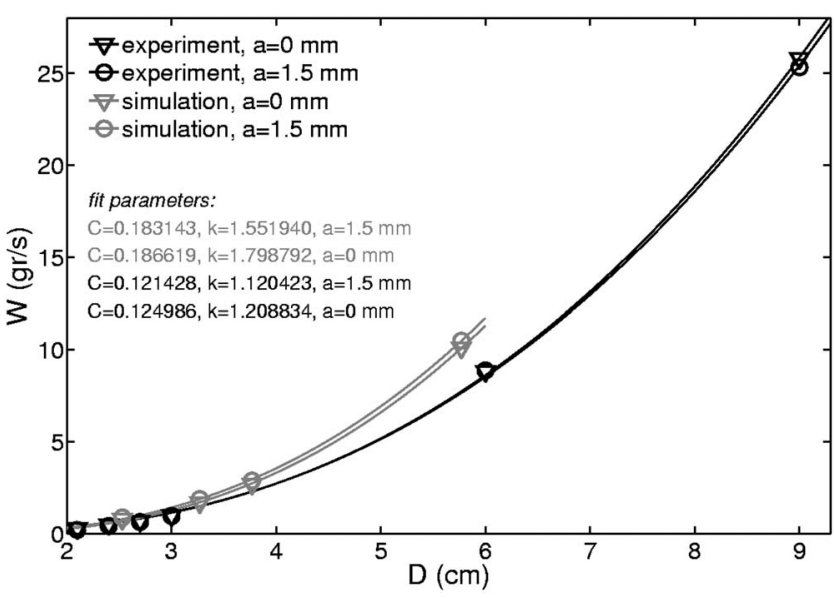

FIG. 4. Flow rates plotted as a function of the outlet diameter. The black markers indicate the results obtained from the experiments and the gray markers indicate the results obtained from the simulation, both for static and vibrating walls $(f=20 \mathrm{~Hz}, a$ $=1.5 \mathrm{~mm}$ ). The solid lines are curves fitted with Beverloo's model. The parameters used are shown in the body of the figure, where $C=\zeta \rho_{B} g^{1 / 2}$.

Air drag, during a silo discharge, is an important issue. It has been shown that an adverse pressure gradient builds up at the bottom of the silo and acts on the particles close to the outlet [17]. However, since the silo is sectioned, there is no pressure gradient built along the column. Thus, air drag cannot explain our findings. In order to explain the above experimental results, we explore the dynamics of the silo discharge with 3D computer simulations.

\section{GRANULAR DYNAMICS SIMULATIONS}

Some computational works have been carried out in the past to study the discharge of silos in two and three dimensions $[18,19]$. Vibrating hoppers have been also investigated [10]. However, we perform simulations in 3D silos with the extra ingredient of having horizontally moving walls. We modified a granular dynamics code previously used in the study of other systems [20].

The particles interact with each other via a soft-sphere model which includes tangential friction. Due to the fact particles in the column are in contact to each other all the time, friction is important to be considered. The system that we studied in the simulations contained 50000 particles with a density of $16 \mathrm{~kg} / \mathrm{m}^{3}$ and an averaged diameter of $4.65 \mathrm{~mm}$. A Gaussian size distribution with a standard deviation of $0.465 \mathrm{~mm}$ was applied to avoid excessive ordering of the particles in the silo. The dimensions of the silo were 0.1 $\times 0.1 \times 0.5 \mathrm{~m}(W \times D \times H)$.

First, the coefficient of restitution was set to the value of 0.9 for the normal direction and 0.33 for the tangential direction. The friction coefficient was set to 0.3. For the particle-wall interaction, the same collision parameters were used. The virtual silo was filled and we plotted the mass transferred to the bottom versus the mass poured inside (light gray line in Fig. 2). Both the mass on the bottom and total 


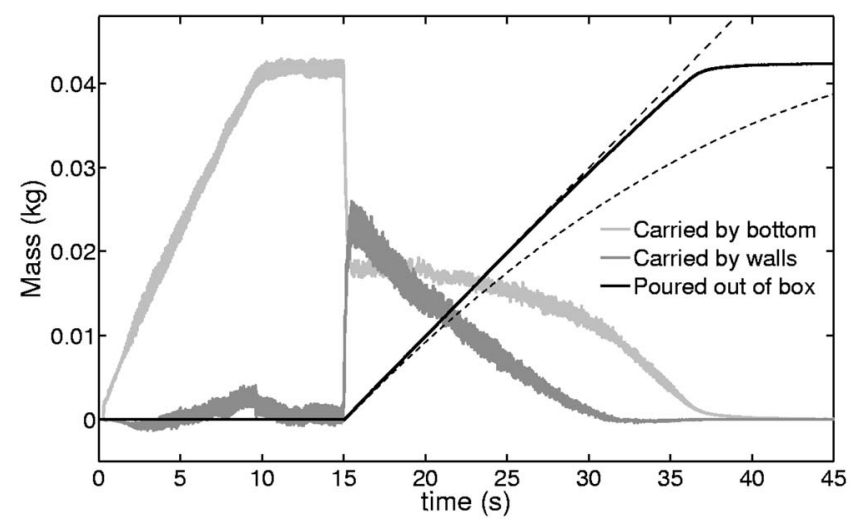

FIG. 5. Mass carried by the bottom (light gray line) and walls (dark gray line) during the discharge. Mass leaving the silo (black line). The vibration conditions are frequency $20 \mathrm{~Hz}$ and amplitude $1.5 \mathrm{~mm}$. The dashed lines resembles Beverloo's and Torricelli's discharge.

mass were multiplied by 5 . Although the light gray line has been scaled in order to compare with the real data, it is obvious that it corresponds to the Janssen's exponential curve. Such result is an indication that the computational code gives the expected arch-shielding behavior found in silos. Thereafter, we filled the silo while the walls were vibrating. As in the real experiment, the result is a straight line (dark gray line in Fig. 2), indicating that hydrostaticlike conditions are obtained.

Next, we perform simulations to analyze the dynamics of the granular flow through openings made in the bottom of the silo for still walls and walls vibrating with a frequency of $20 \mathrm{~Hz}$ and amplitude of $1.5 \mathrm{~mm}$. The results confirm our experimental observations: with or without moving walls, the discharge rates are constant and conform to Beverloo's law when plotted as a function of $D$, see Fig. 4.

In order to understand why the discharge rates are constants despite the column fluidization, we pay attention to the stress distribution during the discharge process. In Fig. 5 we plot the mass carried by the bottom and by the walls before and after the orifice is opened $(t=15 \mathrm{~s})$. Before the discharge (orifice closed), the mass is borne entirely by the bottom, confirming once more the hydrostatic conditions. However, as soon as the hole is opened, the hydrostatic condition is lost and the walls receive part of the weight, reestablishing the Janssen's effect. This may explain why the rates of discharge are constant despite the hydrostatic regime of the column.

We can go beyond our experimental capabilities and investigate computationally what happens if larger shear rates are used. In Fig. 6 we show the results at a higher vibration strength (frequency $50 \mathrm{~Hz}$ and amplitude $3 \mathrm{~mm}$ ). Here, the silo discharges differently: the walls never recover weight and the discharge is not constant, following a Torricelli-like behavior (see how the plot of the mass poured out of the silo bends). It could be argued that the complete absence of wall friction (due to vibration) is responsible for this behavior. However, in Fig. 7 we show simulations for a still silo with frictionless walls and clearly observe that the discharge deviates only slightly from a straight line.

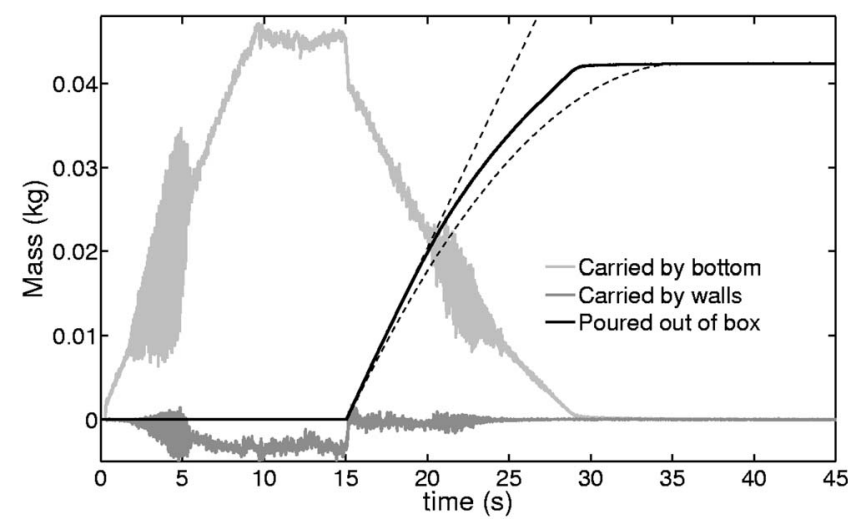

FIG. 6. Mass carried by the bottom (light gray line) and walls (dark gray line) during the discharge. Mass leaving the silo (black line). The vibration conditions are: frequency $50 \mathrm{~Hz}$ and amplitude $3 \mathrm{~mm}$. The dashed lines resembles Beverloo's and Torricelli's discharge.

Figure 8(a) shows a cross section of the granular column with walls vibrating at a frequency of $20 \mathrm{~Hz}$ and amplitude of $3 \mathrm{~mm}$. The color in the beads indicates the force chains distribution. Although a rigorous analysis of force chains is beyond this study, it is illustrative to show how these chains form each vibration cycle. Figures 8(b) and 8(c) show cross sections of the granular column with walls vibrating at a frequency of $50 \mathrm{~Hz}$ and amplitude of $3 \mathrm{~mm}$ at two instants during a vibration cycle. In (b), the column is detached from the wall. This is caused by the large acceleration of the walls; when two walls move inward, the granular mass can either move outward or upward. When the pressure needed to accelerate the particles outwards is larger than the pressure of to the particles above, the particles cannot follow the walls anymore but will move upward instead, and the column detaches from the wall. This is also the reason why with vibrating walls, a bulge is formed on the free surface of the granular mass. The closure of the gap causes a shockwave through the column, as can be seen in (c). However, this shockwave does not influence the outflow rate. A movie of these simulations can be seen here [21].

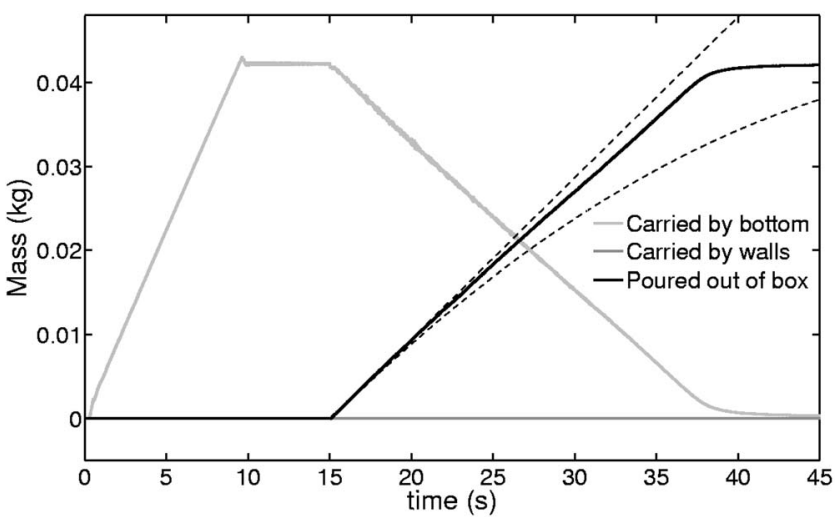

FIG. 7. Silo with frictionless walls. Mass carried by the bottom (light gray line) and walls (dark gray line) during the discharge. Mass leaving the silo (black line). The dashed line resembles Beverloo's and Torricelli's discharge. 

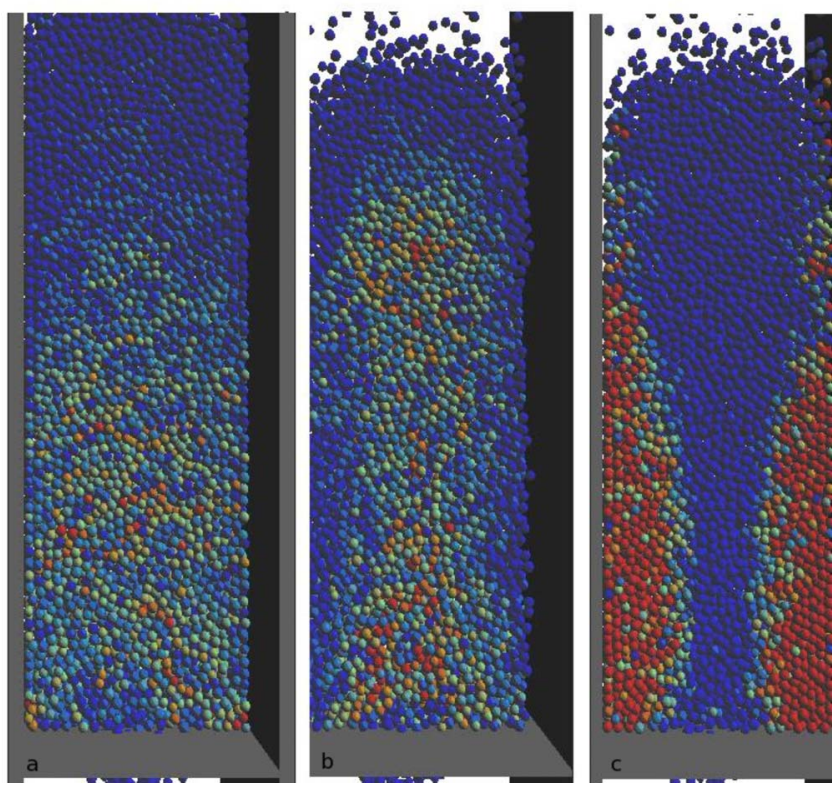

FIG. 8. (Color online) (a) Cross section of the granular column with walls vibrating at a frequency of $20 \mathrm{~Hz}$ and amplitude of $3 \mathrm{~mm}$. The color indicates the force on the particles. Force chains can be identified. (b) and (c) Cross section of the granular column with walls vibrating at a frequency of $50 \mathrm{~Hz}$ and amplitude of $3 \mathrm{~mm}$ at two instances during a vibration cycle. In (b), the column is detached from the wall. The closure of the gap causes a shockwave through the column, as can be seen in (c). However, this shockwave does not influence the outflow rate since this rate scales with the shear rate. A movie of these simulations can be seen in Ref. [21].

As the walls of the silo move in and out, the granular column is sheared with a shear rate approximately four times the velocity of the wall divided by the width of the silo. At a frequency of $20 \mathrm{~Hz}$ and amplitude of $1.5 \mathrm{~mm}$, this wall induced shear rate is around $8 \mathrm{~s}^{-1}$. However, there is also a gravity induced shear rate due to the discharge of the particles. This gravity induced shear rate is approximately the outflow velocity divided by the radius of the aperture, around $30 \mathrm{~s}^{-1}$, i.e., higher than the wall induced shear rate. At a frequency of $50 \mathrm{~Hz}$ and amplitude $3 \mathrm{~mm}$, however, the wall induced shear rate increases to $38 \mathrm{~s}^{-1}$. Is this shear rate increase what causes the Torricelli-like behavior observed in Fig. 6?

Figure 9 shows the averaged (over 15 cycles) flow rates as a function of the pressure on the bottom of the silo for different cases. We also carried out one simulation in a box with dimensions of $0.15 \times 0.15 \times 0.75 \mathrm{~m}(W \times D \times H)$ (i.e., 1.5 times larger than the box used in the former simulation) and 2.25 times as many particles at a frequency of $50 \mathrm{~Hz}$ and an amplitude of $2 \mathrm{~mm}$. The flow rate in this box is lower than the flow rate in the smaller box vibrating with the same frequency and amplitude. We observe that the only two sets of data showing Torricelli-like behavior are the ones obtained at $50 \mathrm{~Hz}$ with amplitudes 2 and $3 \mathrm{~mm}$. This is more evident after rescaling the outflow rate (by subtracting the constant outflow velocity with static walls and dividing by the walls induced shear rate, see Fig. 10). Although the tran-

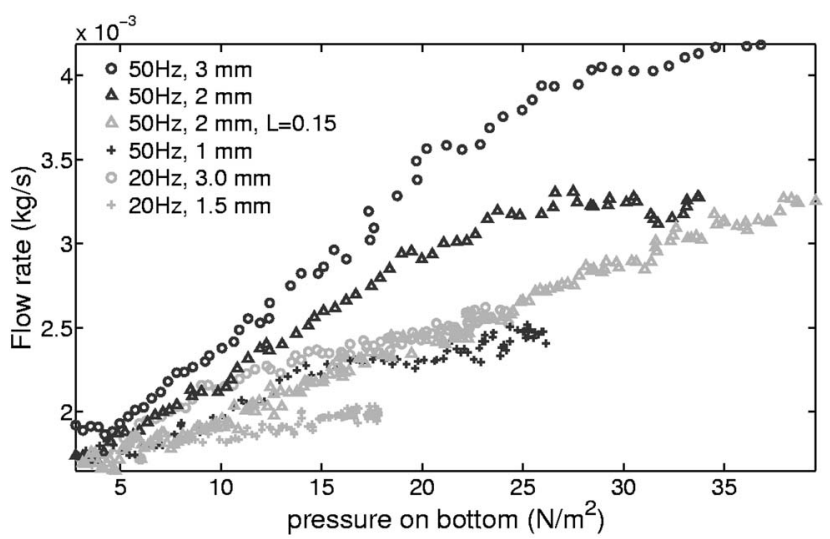

FIG. 9. Flow rates plotted as a function of the pressure on the bottom. All the results are obtained in simulations using a box with a depth and width of $0.1 \mathrm{~m}$, except the dark gray triangles, for which a box with a depth and width of $0.15 \mathrm{~m}$ was used. Note that, with the same vibration parameters, the flow rate in the large box is lower than the flow rate in the standard box. The restitution and friction coefficients are, respectively, 0.9 and 0.3 .

sition between low and high shear regimes is gradual, the change on the silo discharge produced by a high shear is very clear.

Finally, we investigate the effect produced on the discharges when both the restitution and friction coefficients are changed. Values as low as 0.70 for the first one, and as high as 0.50 for the second, were used. The discharge rates (averaged over 15 cycles) do not change much and keep scaling reasonably well (Figs. 11 and 12). The tangential restitution coefficient has also been changed, but no significant sensitivity has been found with this parameter.

\section{CONCLUSIONS}

We have carried out experimental and computational work to study the discharge of a fluidized granular column in a silo with moving walls. We have shown that, at intermedi-

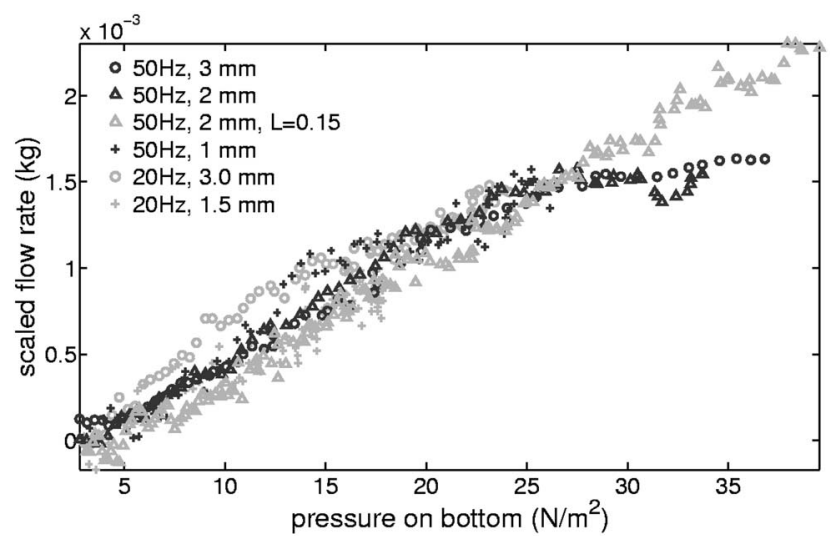

FIG. 10. Scaled flow rates plotted as a function of the pressure on the bottom. The flow rates from Fig. 9 are scaled by subtracting the constant flow rate with static walls divided by the walls induced shear rate. The data collapses reasonably well for low and high shear rates, indicating that the flow rate scales with the wall induced shear rate. 


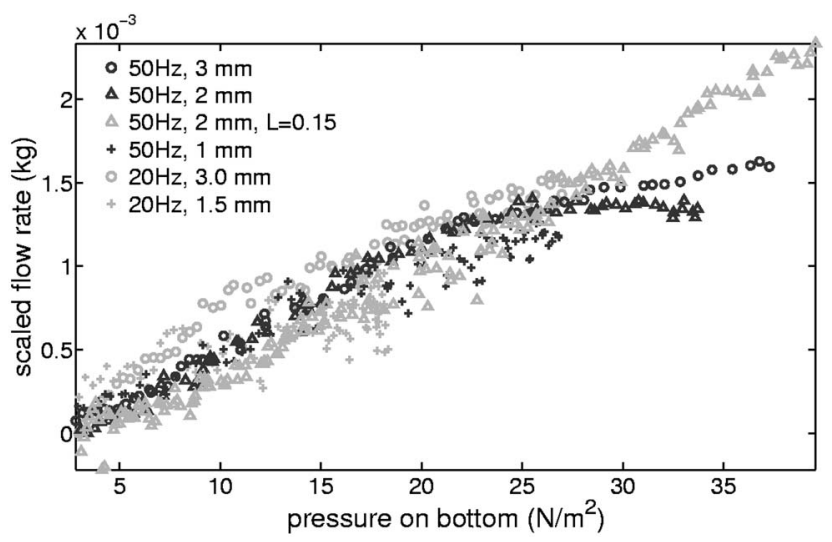

FIG. 11. Scaled flow rates plotted as a function of the pressure on the bottom. The restitution and friction coefficients are, respectively, 0.7 and 0.3 .

ate shear rates, the column attains complete fluidization: the Janssen's screening effect disappears, the pressure becomes linear, and a clear buoyancy effect on light intruders is observed. However, despite the fluidized condition of the column, upon discharge, the screening is recovered (the hydrostaticlike condition is lost) and the discharge rate of the silo is constant. Higher shear rates are necessary to fully suppress screening and observe Torricelli-like discharge rates.

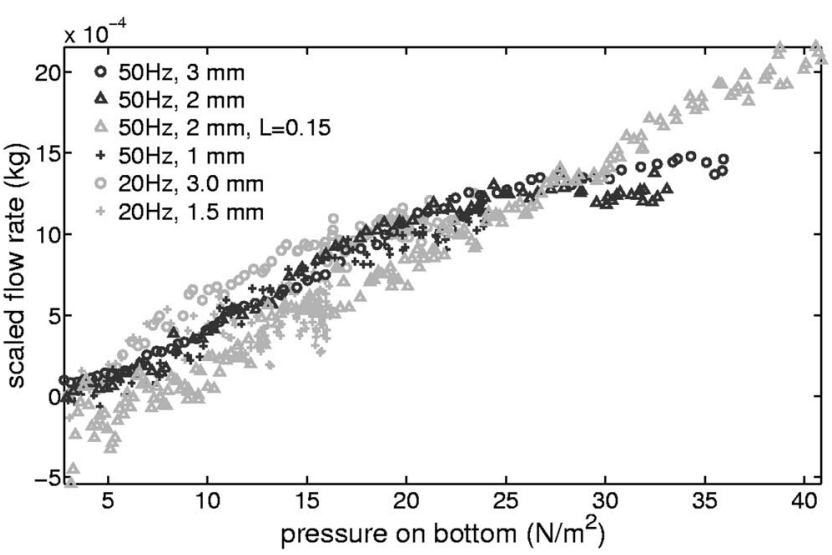

FIG. 12. Scaled flow rates plotted as a function of the pressure on the bottom. The restitution and friction coefficients are, respectively, 0.9 and 0.5 .

\section{ACKNOWLEDGMENTS}

This work has been partially supported by Conacyt, Mexico, under Grant No. 46709. H.P.M. and H.J.v.G. wish to acknowledge support from Conacyt, Mexico and Stichting FOM, respectively. For the numerical simulations we have used the code developed by the group of J.A.M. Kuipers at Twente University. We thank him for allowing us to use his code.
[1] H. A. Janssen, Z. Ver Dtsch. Ing. 39, 1045 (1895).

[2] I. Zuriguel, L. A. Pugnaloni, A. Garcimartin, and D. Maza, Phys. Rev. E 68, 030301(R) (2003).

[3] I. Zuriguel, A. Garcimartin, D. Maza, L. A. Pugnaloni, and J. M. Pastor, Phys. Rev. E 71, 051303 (2005).

[4] L. Vanel, P. Claudin, J. P. Bouchaud, M. E. Cates, E. Clement, and J. P. Wittmer, Phys. Rev. Lett. 84, 1439 (2000).

[5] Y. Bertho, F. Giorgiutti-Dauphine, and J. P. Hulin, Phys. Rev. Lett. 90, 144301 (2003).

[6] R. M. Nedderman, U. Tzn, S. B. Savage, and G. T. Houlsby, Chem. Eng. Sci. 37, 1597 (1982).

[7] W. A. Beverloo, H. A. Leniger, and J. Van de Velde, Chem. Eng. Sci. 15, 260 (1961).

[8] D. Ertas and T. C. Halsey, Europhys. Lett. 60, 931 (2002).

[9] M. L. Hunt et al., Phys. Fluids 11, 68 (1999).

[10] C. R. Wassgren et al., Phys. Fluids 14, 3439 (2002).

[11] K. Chen, M. B. Stone, R. Barry, M. Lohr, W. McConville, K. Klein, B. L. Sheu, A. J. Morss, T. Scheidemantel, and P. Schiffer, Phys. Rev. E 74, 011306 (2006).

[12] C. T. Veje and P. Dimon, Granular Matter 3, 151 (2004).

[13] D. A. Huerta, V. Sosa, M. C. Vargas, and J. C. Ruiz-Suarez, Phys. Rev. E 72, 031307 (2005).
[14] J. B. Knight, H. M. Jaeger, and S. R. Nagel, Phys. Rev. Lett. 70, 3728 (1993).

[15] M. E. Mobius, X. Cheng, G. S. Karczmar, S. R. Nagel, and H. M. Jaeger, Phys. Rev. Lett. 93, 198001 (2004).

[16] Y. Nahmad-Molinari, G. Canul-Chay, and J. C. Ruiz-Suarez, Phys. Rev. E 68, 041301 (2003).

[17] B. J. Crewdson, A. L. Ormond, and R. M. Nedderman, Powder Technol. 21, 245 (1977).

[18] D. Hirshfeld, Y. Radzyner and D. C. Rapaport, Phys. Rev. E 56, 4404 (1997).

[19] D. Hirshfeld and D. C. Rapaport, Eur. Phys. J. E 4, 193 (2001).

[20] M. A. van der Hoef, M. Ye, M. van Sint Annaland, A. T. Andrews IV, S. Sundaresan, and J. A. M. Kuipers, Adv. Chem. Eng. 31, 65 (2006); H. J. van Gerner, M. A. van der Hoef, D. van der Meer, and K. van der Weele, Phys. Rev. E 76, 051305 (2007).

[21] See EPAPS Document No. E-PLEEE8-77-116802 for a video of the simulations with walls vibrating at 20 and $50 \mathrm{~Hz}$. For more information on EPAPs, see http://www.aip.org/pubservs/ epaps.html. 